\title{
May Smoking and Alcohol Consumption Worsen the Spermiogram Results?
}

\author{
Hatem KAZIMOGLU1, Yunus Emre TOPDAGI², Mehmet SOLAKHAN ${ }^{3}$, Ali Irfan GUZEL² \\ Gaziantep, Turkey
}

\section{ABSTRACT}

OBJECTIVE: In recent years, the number of infertile couples who desire pregnancy with assisted reproduction techniques is increasing. Smoking and alcohol consumption are important factors affecting the treatment of fertility and assisted reproductive techniques. To evaluate the effect of smoking and alcohol consumption on spermiogram results

STUDY DESIGN: This prospective case-control study was conducted at current urology and infertility department in a tertiary research hospital and a total of 6171 cases included in the study. Data collected and evaluated were age and sperm parameters (liquefaction, semen volume, sperm concentration, total number, total motility, progressive motility, slow motility, non-progressive motility, morphology).

RESULTS: Of 6171 patients; a total of 3247 men was smoker ( $\mathrm{n}: 3247, \% 52.6)$ and 3511 was alcohol users ( $\mathrm{n}: 3511, \% 56.9$ ). Mean age of the patients in the study group was $32.8 \pm 6.5$ years. There was a statistically significant difference between the smoker and nonsmoker in terms of sperm concentration and slow motility $(p<0.05)$. There was a statistically significant difference between alcohol consumption and no alcohol consumption in terms of; semen volume, sperm concentration, normal morphology $(p<0.05)$.

CONCLUSION: According to this study, smoking has a negative effect on sperm concentration and slow motility. Alcohol consumption has a negative impact on semen volume, sperm concentration, normal morphology. Smoking and alcohol consumption separately and combined were found to have a deleterious effect on sperm parameters. It is suggested that both habits may contribute to infertility problems.

Keywords: Alcohol, Male infertility, Smoking, Spermiogram

Gynecol Obstet Reprod Med 2021;27(1):44-48

\section{Introduction}

Infertility; with a ratio of $15 \%$ worldwide, is defined as the inability to achieve pregnancy despite 1 year of regular and unprotected sexual intercourse by the American Society for Reproductive Medicine (ASRM) (1,2). Infertile couples have

I Sanko University Medical Faculty Department of Urology, Gaziantep,

2 Sanko University Medical Faculty Department of Obstetrics and Gynecology, Gaziantep, Turkey

3 Bahcesehir University Medical Faculty Department of Urology, İstanbul,

Address of Correspondence: Yunus Emre Topdagi

Sanko University School of Medicine,

Department of Gynecology and Obstetrics,

27090, Gaziantep, Turkey

emr-topdagi@hotmail.com

Submitted for:Publication 09.07.2019

Revised for: Publication 27.08.2019

Accepted for Publication: $\quad$ 23.09.2019

ORCID IDs of the authors: HK: 0000-0001-8224-5068,

YET: 0000-0003-0656-0765 MS: 0000-0001-9123-9196,

AIG: 0000-0002-9518-3772

\begin{tabular}{|c|c|}
\hline \multirow{3}{*}{ 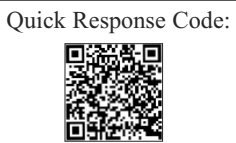 } & Access this article online \\
\hline & $\begin{array}{l}\text { Website: www.gorm.com.tr } \\
\text { e- mail: info@gorm.com.tr }\end{array}$ \\
\hline & DOI:10.21613/GORM.2019.1001 \\
\hline
\end{tabular}

How to cite this article: Kazlmoglu H. Topdagi YE. Solakhan M. Guzel AI. May Smoking and Alcohol Consumption Worsen the Spermiogram Results? Gynecol Obstet Reprod Med 2021;27(1):44-48
$20 \%$ male and female factors and only male factor in $25 \%$ of all couples with infertility (3). Smoking and alcohol have a negative influence on testicular and ovarian functions, calcium metabolism, and the activity of insulin (4). In the USA the ratio of alcohol use was reported to be $70.7 \%$ in the past year and $56 \%$ in the previous month (5). Alcohol is a known teratogen, but its relation to the amount of consumption and the risk of infertility is not clear. Excessive alcohol use causes testicular atrophy, impotence, decreased libido and decreased sperm count (6). The relationship between alcohol consumption and reduced semen quality depends on both the metabolism of testosterone and the direct negative effects on spermatogenesis (7).

In previous studies, researchers reported that there was a negative association between alcohol consumption, smoking and semen quality $(8,9)$. In many studies, smoking was found to be effective on sperm quality such as semen volume, sperm concentration, motility and morphology (10).

In this study, we aimed to demonstrate the potential effects of smoking and alcohol consumption on male infertility.

\section{Material and method}

This prospective case-control study was designed at Sanko University, School of Medicine, Department of Urology and 
Infertility, from 2010 to 2017 . The study was approved by the Institutional Review Board (Approval number: 2018/11-10). The study was conducted in accordance with the Declaration of Helsinki. Ethics committee approval was received for this study from the ethics committee of Gaziantep University. This is a tertiary research and education hospital in Gaziantep.

A total of 6171 men with infertility were undergone spermiogram. 3247 men were smokers and 3511 was alcohol users. The analysis was performed by dividing the cases into two groups as; smokers (n:3247, 52.6\%) vs non-smokers (n:2924, 47.4\%) and alcohol users (n:3511, 56.9\%) vs nonusers (n:2660, 43.1\%). The parameters evaluated were; age and sperm parameters (liquefaction, semen volume, sperm concentration, total number, total motility, progressive motility, slow motility, non-progressive motility, morphology).

The daily smoking amount was recorded at the package level. They were questioned whether they used alcohol was recorded without quantity. The yearly calculation was calculated as package/year. The total number of packets/years they use is specified. Data recorded for each patient were obtained from the patients' files and hospital database.

Semen samples were obtained by masturbation in sterile containers after at least three days of sexual abstinence. For the first macroscopic examination, semen samples were expected to be liquefied at $37{ }^{\circ} \mathrm{C}$ between $20-40$ minutes. The semen, whose viscosity, appearance, volume, $\mathrm{pH}$, liquefaction, concentration, total number, motility, morphology was evaluated, was examined by light microscopy with X100 magnification for microscopic evaluation. It was analyzed in accordance with the WHO-2010 (11).

\section{Statistics}

The normality of distribution of continuous variables was tested by the Shapiro Wilk test. Kruskal Wallis and Dunn multiple comparison tests were used to comparing 3 independent groups for nonnormal data. Chi-square test was used to investigate the relationship between two categorical variables and Bonferroni correction was applied to adjust $\mathrm{p}$ values for multiple comparisons when Chi-square test result is significant. To decrease bias due to the small prevalence's of same categories in binomial-response general linear model estimates Firth lo- gistic regression model was applied in brglm package in $\mathrm{R}$ version 3.5.1. Multiple linear regression analysis was performed to determinate impact on smoking, alcohol consumption on parameters after adjusting age. ROC curve analysis was used to evaluate whether there is a significant cut-off value for alcohol consumers and smokers or not. All univariate statistical analysis was performed with SPSS for Windows version 24.0 and a $p$ value $<0.05$ was accepted as statistically significant.

\section{Results}

In the current study, we evaluated the sperm parameters of 6171 men applied to our infertility clinic. We designed a prospective case-control study; a total of 3247 men was smokers (n:3247, \%52.6) and 3511 were alcohol users (n:3511, \%56.9). Table I demonstrated the distribution of ages and spermiogram results according to the years. The demographic and clinical characteristics of the patients are shown in table II. Mean age of the patients in the study group was $32.8 \pm 6.5$ years. There was a statistically significant difference between smoker and non-smoker in terms of sperm concentration and slow motility $(p<0.05)$. The sperm concentration was significantly lower in smokers. Slow motility of sperm parameter was significantly higher in the smoker group. There was a statistically significant difference between alcohol consumption and non-alcohol consumption in terms of; semen volume, sperm concentration, normal morphology $(p<0.05)$. Semen volume was significantly higher in the group of alcohol consumption. The sperm concentration and normal morphology were significantly lower in alcohol users.

Table III summarizes the outcomes of Logistic Regression Models. According to the model smoking has a negative effect on sperm concentration, total motility, progressive motility and slow motility with odds ratio of $6.4,5.6,5.4$ and 7.3; respectively $(p<0.05)$, and alcohol has a negative effect on sperm concentration, total motility and slow motility with odds ratio of 5.9, 3.9 and 6; respectively $(p<0.05)$.

ROC curve analysis demonstrated the AUC for age, sperm concentration, total motility, slow motility, morphology and depicted the area under the curve (AUC), cut off value and sensitivity and specificity of these variables (Figure 1).

Table I: Distribution of spermiogram results according to the years

\begin{tabular}{lccccc}
\hline Years & $\begin{array}{c}\text { Age } \\
\text { (years })\end{array}$ & $\begin{array}{c}\text { Liquefaction } \\
(\mathrm{min})\end{array}$ & $\begin{array}{c}\text { Volume } \\
(\mathrm{mL})\end{array}$ & $\begin{array}{c}\text { Sperm concentration } \\
(\mathrm{M} / \mathrm{mL})\end{array}$ & $\begin{array}{c}\text { Total number } \\
(\mathrm{M})\end{array}$ \\
\hline $2010(\mathrm{n}=802)$ & $35.07 \pm 6.21$ & $22.23 \pm 7.82$ & $2.61 \pm 1.13$ & $64.87 \pm 134.19$ & $102.92 \pm 124.61$ \\
$2011(\mathrm{n}=751)$ & $34.69 \pm 6.37$ & $22.01 \pm 9.65$ & $2.61 \pm 1.17$ & $36.52 \pm 55.03$ & $87.35 \pm 91.22$ \\
$2012(\mathrm{n}=679)$ & $34.17 \pm 6.11$ & $21.88 \pm 9.41$ & $2.21 \pm 1.13$ & $30.99 \pm 24.59$ & $67.1 \pm 62.82$ \\
$2013(\mathrm{n}=671)$ & $33.4 \pm 6.07$ & $22.82 \pm 11.25$ & $2.27 \pm 1.16$ & $28.26 \pm 24.36$ & $60.3 \pm 59.54$ \\
$2014(\mathrm{n}=818)$ & $32.61 \pm 6.92$ & $23.6 \pm 13.16$ & $2.98 \pm 1.56$ & $35.27 \pm 31.69$ & $98.03 \pm 99.94$ \\
$2015(\mathrm{n}=609)$ & $31.82 \pm 6.37$ & $27.87 \pm 14.93$ & $3.6 \pm 1.63$ & $50.9 \pm 45.64$ & $167.65 \pm 162.78$ \\
$2016(\mathrm{n}=887)$ & $31.14 \pm 6.49$ & $27.74 \pm 14.25$ & $3.71 \pm 1.99$ & $48.62 \pm 63.19$ & $166.48 \pm 173.92$ \\
$2017(\mathrm{n}=954)$ & $30.34 \pm 6.26$ & $28.58 \pm 14.84$ & $3.91 \pm 1.73$ & $43.79 \pm 43.47$ & $163.66 \pm 168.67$ \\
\hline
\end{tabular}


Table II: Demographic and clinical characteristics of the patients

\begin{tabular}{|c|c|c|c|}
\hline $\begin{array}{l}\text { Variable groups } \\
\text { smoking }\end{array}$ & $\begin{array}{c}\text { User } \\
(n=3247)\end{array}$ & $\begin{array}{l}\text { Nonuser } \\
(n=2924)\end{array}$ & $p$ \\
\hline Liquefaction (min) & $24.74 \pm 12.64$ & $24.74 \pm 12.53$ & 0.976 \\
\hline Semen volume $(\mathrm{mL})$ & $3.06 \pm 1.69$ & $3 \pm 1.55$ & 0.132 \\
\hline Sperm concentration $(\mathrm{M} / \mathrm{mL})$ & $40.29 \pm 52.5$ & $45.62 \pm 75.61$ & $0.001^{*}$ \\
\hline Total number (M) & $114.58 \pm 129.37$ & $119.08 \pm 141.25$ & 0.192 \\
\hline Total motility (\%) & $43.41 \pm 21.77$ & $42.63 \pm 22.3$ & 0.164 \\
\hline Prog. Motility (M) & $29.67 \pm 18.39$ & $29.13 \pm 19$ & 0.257 \\
\hline Slow motility (M) & $7.23 \pm 5.6$ & $6.87 \pm 5.65$ & $0.013^{*}$ \\
\hline Non progressive motility (M) & $6.34 \pm 4.53$ & $6.14 \pm 4.72$ & 0.101 \\
\hline N. Morphology \% & $11.99 \pm 6.71$ & $11.69 \pm 7.04$ & 0.091 \\
\hline Alcohol consumption & $(n=3511)$ & $(n=2660)$ & \\
\hline Liquefaction (min) & $24.95 \pm 12.64$ & $24.46 \pm 12.53$ & 0.126 \\
\hline Semen volume $(\mathrm{mL})$ & $3.08 \pm 1.69$ & $2.97 \pm 1.55$ & $0.009^{*}$ \\
\hline Sperm concentration $(\mathrm{M} / \mathrm{mL})$ & $40.51 \pm 52.5$ & $45.86 \pm 75.61$ & $0.001^{*}$ \\
\hline Total number (M) & $115.55 \pm 129.37$ & $118.25 \pm 141.25$ & 0.436 \\
\hline Total motility (\%) & $42.63 \pm 21.77$ & $43.59 \pm 22.3$ & 0.087 \\
\hline Prog. Motility (M) & $29.19 \pm 18.39$ & $29.71 \pm 19$ & 0.282 \\
\hline Slow motility (M) & $7.04 \pm 5.6$ & $7.09 \pm 5.65$ & 0.737 \\
\hline Non progressive motility (M) & $6.16 \pm 4.53$ & $6.36 \pm 4.72$ & 0.093 \\
\hline N. Morphology (\%) & $11.68 \pm 6.71$ & $12.08 \pm 7.04$ & $0.023^{*}$ \\
\hline
\end{tabular}

${ }^{*} p<0.05$ : Two-sided $p$ values were considered statistically significant. Prog: Progressive, $N$ : Normal

Table III: The outcomes of logistic regression models

\begin{tabular}{lcccc}
\hline & \multicolumn{2}{c}{ Smoking } & \multicolumn{2}{c}{ Alcohol consumption } \\
\cline { 2 - 5 } & Odds ratio & $p$ & Odds ratio & $p$ \\
\hline Liquefaction (min) & .021 & .884 & 1.077 & .299 \\
Semen volume (ml) & 3.266 & .071 & 3.738 & .053 \\
Sperm concentration (106 /ml) & 6.400 & .011 & 5.972 & .015 \\
Total number (M) & .310 & .578 & .000 & .982 \\
Total motility (\%) & 5.698 & .017 & 3.955 & .047 \\
Prog. Motility (M) & 5.481 & .019 & 3.499 & .061 \\
Slow motility (M) & 7.373 & .007 & 6.071 & .014 \\
Non progressive motility (M) & 1.399 & .237 & .114 & .736 \\
N. Morphology (\%) & 1.439 & .230 & .498 & .481 \\
\hline
\end{tabular}

*Significant at 0.05 level, Wald test. Prog: progressive, N: Normal

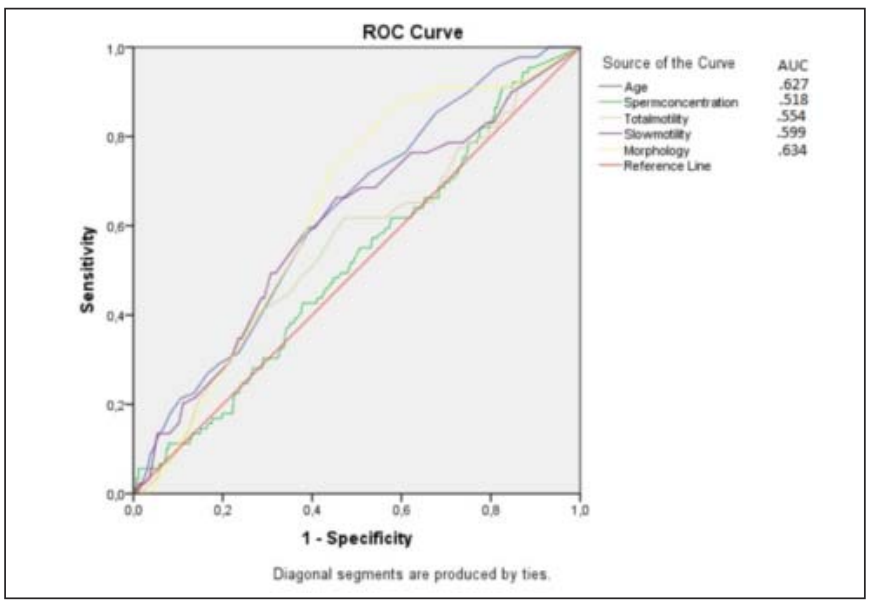

Figure 1: ROC curve analysis

\section{Discussion}

In the current study, we evaluated the effects of smoking and alcohol consumption on sperm results in infertile men. There was a statistically significant difference between smoker and non-smokers in terms of sperm concentration and slow motility $(p<0.05)$. There was a statistically significant difference between alcohol consumption and non-alcohol consumption in terms of; semen volume, sperm concentration, normal morphology $(p<0.05)$.

The relationship between impaired reproductive functions and smoking and alcohol use is suspected. Many studies have shown the negative effects of smoking and alcohol use on male fertility. Smoking has been shown to have a detrimental effect on various parameters of semen analysis. These nega- 
tive effects are about sperm production, motility, and morphology (12). Analysis of 27 studies in smokers and semen quality showed a $13 \%$ decrease in sperm concentration, $10 \%$ in sperm motility and a 3\% decrease in normal sperm morphology in smokers (13). To better understand the effect of the number of smoked cigarettes per day on semen quality, we recorded the number of cigarettes. A cross-sectional analysis of 2542 healthy men found that on semen analysis, cigarette smokers had lower semen volumes, sperm counts and percentage of motile sperm compared to men who did not smoke (12). In addition, it has been suggested that the relationship between smoking and sperm concentration is dependent on the duration of use. Similar results were obtained in our study.

In a large cohort of 1786 men with infertility showed that smoking was associated with a decrease in sperm density $(15.3 \%)$, total sperm counts $(17.5 \%)$, and total motile sperm (16.6\%) compared with nonsmokers. In addition, morphology was slightly affected by smoking, but not significantly (14). In another study, 362 Chinese men with infertility found that smokers demonstrated decreased semen volumes, sperm concentrations (15). Another study of 200 infertile men found higher rates of decreased sperm motility and abnormal sperm morphology among smokers (16).

In other cohort studies, low sperm concentrations and higher abnormal sperm morphology rates were found to be dose-dependent in smokers (17). For this reason, the number of cigarette smokers seemed to be at risk for the negative effects on fertility. Some studies have shown that smoking is not an independent risk factor for decreased motile sperm concentrations. In another study, it was observed that there was no significant risk factor for semen quality among the 626 men who applied to infertility clinics, neither smoking nor chewing tobacco (18).

There are very few meta-analyses to examine the relationship between smoking and semen parameters. In a meta-analysis study, smoking had negative effects on all sperm parameters including semen volume, sperm density, total sperm count and percentage of sperm with progressive mobility (19). In another meta-analysis study, it was found that smokers had $13 \%$ to $17 \%$ lower sperm density than non-smokers (20).

Some studies also showed a negative relationship between alcohol consumption and semen quality (8). Some authors have not found this relationship (7). In a historical study of 685 men who drank alcohol (beer and wine) systematically over a period of 30-60 minutes, delayed seminal fluid liquefaction associated with low sperm motility was detected (21). A significant seminal fluid volume and decreased sperm concentration have been reported in 20 men with alcohol dependence syndrome. In addition, there was a higher rate of morphologically abnormal sperm in these men compared to controls, but no correlation was found with the amount or duration of alcohol consumption (22). In addition, most studies have only addressed average alcohol intake by asking only a few questions, and it is likely that consumption within the response categories can vary considerably and be reported low.

On the other hand, it was reported that sperm parameter abnormalities were significantly associated with high serum LH, FSH, and 17beta-estradiol levels and significantly decreased with serum testosterone levels and thus, the presence of primary testiculopathy in men drinking ethanol reveals (23). Goverde et al. did not find any statistically significant difference for seminal fluid volüme sperm concentration and percentage of motile spermatozoa in daily drinkers and subfertile patients (24).

Gaur et al. reported that only $12(100 \%)$ of 100 alcoholics had normozoospermia, and 37 (37\%) of the 100 nonalcoholic control groups (8). A study of 1221 young Danish males found that sperm concentration and total sperm count were negatively correlated with increased alcohol consumption (25). A casecontrol study concluded that the volume of semen and sperm concentration in alcoholics was lower than abstainers (23). The discrepancy between our findings and our previous studies may be due to the different categorization of alcohol consumption and different drinking habits of studied populations.

On the other hand, different studies have proved experimentally that alcohol has a detrimental effect on all levels of the male reproductive system. Alcohol interferes with the function of the hypothalamic pituitary testicular axis, impairing gonadotropin secretion with consequent decreasing of testosterone levels $(23,26)$. In general, the studies presented in this review show that alcohol consumption changes sperm parameters. Finally, nutritional deficiency, as well as genetic history, may alter the effect of alcohol on spermatogenesis.

As a result, we found that semen quality did not become worse with occasional alcohol consumption, whereas both volume and morphology were adversely affected by daily consumption. In addition, well-designed studies describing the categories of alcohol consumption as well as predefined criteria for selecting subjects are essential to obtain good evidence about the effect of alcohol on semen parameters. There is a need for studies evaluating the effect of changes in sperm parameters on reproductive outcomes in order to make recommendations about alcohol consumption other than advice to avoid heavy alcoholic beverages. Therefore, it should be advised that men with fertility difficulties should stop smoking and alcohol consumption as soon as possible to optimize their reproductive potential. Finally, cigarette smoking affects semen analysis outcomes in infertile men.

Acknowledgments: None

Author Contributions: Concept: HK,YET. Design:HK,MS. Supervision:YET, HK, MS, Resources: HK,YET. Materials: YET,HK,MS. Data Collection and Processing:HK,YET, Analysis and Interpretation:HK,YET,AIG.Literature Search: YET, AIG, Writing Manuscript: HK,YET. Critical Review: YET,AIG. Funding: None. Conflict of interest: None 


\section{References}

1. Practice Committee of American Society for Reproductive Medicine. Definitions of infertility and recurrent pregnancy loss: a committee opinion. Fertil Steril. 2013;99(1):63. doi: 10.1016/j.fertnstert.2012.09.023.

2. Thoma ME. McLain AC. Louis JF. et al. Prevalence of infertility in the United States as estimated by the current duration approach and a traditional constructed approach. Fertil Steril. 2013;99(5):1324-1331.e1321. doi:10.1016/j. fertnstert.2012.09.023.

3. Kovac JR. Pastuszak AW. Lamb DJ. The use of genomics, proteomics, and metabolomics in identifying biomarkers of male infertility. Fertil Steril. 2013;99(4):998-1007. doi: 10.1016/j.fertnstert.2013.01.111.

4. Starka L. Hill M. Kralikova E. Smoking and endocrine system (in Czech). DMEV. 2005;8:180-6.

5. Ricci E. Al Beitawi S. Cipriani S. Candiani M. Chiaffarino F. Viganò P. Noli S. Parazzini F. Semen quality and alcohol intake: a systematic review and meta-analysis. Reprod Biomed Online. 2017;34(1):38-47. doi:10.1016/j.rbmo. 2016.09.012.

6. Pramanic P. Impact of adulthood lifestyle on male infertility: A critical review of the current literature. Int J Life Sci Pharm Res. 2012;2(4):51-61.

7. Hansen ML. Thulstrup AM. Bonde JP. Olsen J. Håkonsen LB. Ramlau-Hansen CH. Does last week's alcohol intake affect semen quality or reproductive hormones? A crosssectional study among healthy young Danish men. Reprod Toxicol. 2012;34:457-62. doi:10.1016/j.reprotox.2012. 06.004 .

8. Gaur DS. Talekan MS. Pathak VP. Alcohol intake and cigarette smoking: impact of two major lifestyle factors on male fertility. Indian J Pathol Microbiol. 2010;53:35-40. doi:10.4103/03774-929.59180.

9. Jandíková H. Dušková M. Stárka L. The influence of smoking and cessation on the human reproductive hormonal balance. Physiol Res. 2017;66(Supplementum 3): S323-S331. doi: 10.33549/physiolres.933724.

10. Pasqualotto FF. Umezu FM. Salvador M. Borges EJr. Sobreiro BP. Pasqualotto EB. Effect of cigarette smoking on antioxidant levels and presence of leukocytospermia in infertile men: a prospective study. Fertil Steril. 2008; 90:278-28. doi: 10.1016/j.fertnstert.2008.02.123.

11. Cooper TG. Noonan E. Eckardstein S von. et al. World Health Organization reference values for human semen characteristics. Hum Reprod Update 2010;16(3):231-45. doi: 10.1093/humupd/dmp048.

12. Ramlau-Hansen CH. Thulstrup AM. Aggerholm AS. Jensen MS. Toft G. Bonde JP. Is smoking a risk factor for decreased semen quality? A cross-sectional analysis. Hum Reprod. 2007a;22:188-196. doi: 10.1093/humrep/del364.

13. Vine MF. Smoking and male reproduction: a review. Int $\mathbf{J}$
Androl. 1996;19:323-337. doi:10.1111/j.1365-2605.1996. tb00523.x.

14. Kunzle R. Mueller MD. Hanggi W. Birkhauser MH. Drescher H. Bersinger NA. Semen quality of male smokers and nonsmokers in infertile couples. Fertil Steril. 2003; 79(2):287-287. doi: 10.1016/s0015-0282(02)046 64-2.

15. Zhang JP. Meng QY. Wang Q. Zhang LJ. Mao YL. Sun ZX. Effect of smoking on semen quality of infertile men in Shandong, China. Asian J Androl. 2000;2(2):143-6. PMID: 11232793.

16. Gaur DS. Talekar M. Pathak VP. Effect of cigarette smoking on semen quality of infertile men. Singapore Med J. 2007;48(2):119-23. PMID:17304390.

17. Chia SE. Ong CN. Tsakok FM. Effects of cigarette smoking on human semen quality. Arch Androl. 1994; 33(3): 163-8. doi: 10.3109/01485019408987820.

18. Dikshit RK. Buch JG. Mansuri SM. Effect of tobacco consumption on semen quality of a population of hypofertile males. Fertil Steril. 1987;48(2):334-6. doi:10.1016/ s0015-0282(16)59369-8.

19. Li Y. Lin H. Li Y. Cao J. Association between socio-psycho-behavioral factors and male semen quality: systematic review and meta-analyses. Fertil Steril. 2011;95(1):11623. doi: 10.1016/j.fertnstert.2010.06.031.

20. Vine MF. Margolin BH, Morrison HI. Hulka BS. Cigarette smoking and sperm density: a meta-analysis. Fertil Steril. 1994;61(1):35-43. doi:10.1016/S0015-0282 (16)56450-4.

21. Molna'r J. Papp G. Alcohol as a possible stimulant of mucous production in the semen. Andrologie. 1973;5:1056.doi: 10.1111/j.1439-0272.1973.tb00334.x.

22. Kucheria K. Saxena R. Mohan D. Semen analysis in alcohol dependence syndrome. Andrologia. 1985;17(6):55863. doi: 10.1111/j.1439-0272.1985.tb01714.x.

23. Muthusami KR. Chinnaswamy P. Effect of chronic alcoholism on male fertility hormones and semen quality. Fertil Steril 2005;84:919-24. doi: 10.1016/j.fertnstert. 2005.04.025

24. Goverde HJ, Dekker HS, Janssen HJ, Bastiaans BA, Rolland R et al. Semen quality and frequency of smoking and alcohol consumption-an explorative study. Int J Fertil Menopausal Stud 1995;40: 135-8. PMID:7663540.

25. Jensen TK. Gottschau M. Madsen JO. Andersson AM. Lassen TH. Skakkebaek NE. et al. Habitual alcohol consumption associated with reduced semen quality and changes in reproductive hormones; a cross sectional study among 1221 young Danish men'. BMJ Open 4, e005462. doi: 10.1136/bmjopen-2014-005462.

26. Maneesh M. Dutta S. Chakrabarti A. Vasudevan DM. Alcohol abuse-duration dependent decrease in plasma testosterone and antioxidants in males. Indian $\mathrm{J}$ Physiol Pharmacol. 2006;50(3):291-6. PMID:17193902. 